\title{
A BLOCK ARNOLDI BASED METHOD FOR THE SOLUTION OF THE SYLVESTER-OBSERVER EQUATION*
}

\author{
L. ELBOUYAHYAOUI ${ }^{\dagger}$, M. HEYOUNI ${ }^{\ddagger}$, K. JBILOU $^{\S}$, AND A. MESSAOUDI ${ }^{\natural}$
}

\begin{abstract}
This paper describes a new block method for solving multi-input Sylvester-observer equations that arise in the construction of the well-known Luenberger observer. The proposed method is based on the block Arnoldi process and generalizes to the multi-input case, the method proposed by Datta and Saad for the single input Sylvester-observer equation. We give new algebraic properties and show how to construct the Luenberger observer by solving a special large-scale Sylvester equation for which two unknown matrices are to be computed. The numerical tests show that the proposed approach is effective and can be used for large-scale Luenberger observer problems.
\end{abstract}

Key words. Block Arnoldi process, Luenberger-observer, Sylvester equation

AMS subject classifications. $65 \mathrm{~F} 10$

1. Introduction. Consider the Multi-Input Multi-Output (MIMO) linear time-invariant dynamical system

$$
\begin{aligned}
& \dot{\hat{x}}(t)=A^{T} \widehat{x}(t)+B \widehat{u}(t), \quad \widehat{x}(0)=\widehat{x}_{0}, \\
& \widehat{y}(t)=C^{T} \widehat{x}(t), \quad t \geq 0,
\end{aligned}
$$

where $\widehat{x} \in \mathbb{R}^{n}$ is the state vector, $\widehat{y} \in \mathbb{R}^{q}$ is the output vector, and $\widehat{u} \in \mathbb{R}^{p}$ is the input vector. The state matrix $A \in \mathbb{R}^{n \times n}$ is assumed to be large and sparse, $B \in \mathbb{R}^{n \times p}$ and the output matrix $C \in \mathbb{R}^{n \times q}$. Throughout this paper we will assume that $p, q \ll n$.

In many situations the states $\widehat{x}_{0}$ and $\widehat{x}(t)$ are not explicitly known. Thus, as the state of the system is generally not accessible, the objective of an observer is to obtain information about these unknown states. This is done by estimating the state $\widehat{x}(t)$ by another variable denoted by $x(t)$. The most commonly used technique is to design another control system related to the system (1.1) whose entries consist of all the information available and whose output will be precisely $x(t)$. For more details on this subject, we refer to [5, Chapter 12] or [16, Chapter 4] and to the references cited therein.

In [18], Luenberger proposed the construction of an approximation $x(t)$ of the initial state $\widehat{x}(t)$ as follows

$$
\dot{x}(t)=\widehat{H}^{T} x(t)+G^{T} \widehat{y}(t)+X^{T} B \widehat{u}(t), \quad x(0)=x_{0},
$$

where the unknown matrices $\widehat{H}, G \in \mathbb{R}^{q \times q}$ and $X \in \mathbb{R}^{n \times q}$ have to be determined. Luenberger proposed to compute $x(t)$ such that it approximates $X^{T} \widehat{x}(t)$. The system (1.2) is commonly called a Luenberger observer and the matrices $\widehat{H}, X, G$ are to be determined such that

$$
A X-X \widehat{H}=C G \text {. }
$$

*Received December 4, 2016. Accepted May 24, 2017. Published online on September 8, 2017. Recommended by L. Reichel.

${ }^{\dagger}$ Centre Régional des Métiers de l'Education et de la Formation, CRMEF, Fes, Equipe EMMA, Laboratoire LSIA, ENSAH, Morocco (lakhdarr2000@yahoo.fr).

${ }^{\ddagger}$ ENSA d'Al-Hoceima, Equipe EMMA, Laboratoire LSIA, Université Mohammed Premier, Oujda, Morocco (mohammed.heyouni@gmail.com).

$\S$ L.M.P.A, Université du Littoral, 50 rue F. Buisson BP 699, F-62228 Calais Cedex, France (jbiloullmpa.univ-littoral.fr).

"Ecole Normale Supérieure, Mohammed V University in Rabat, Morocco (abderrahim.messaoudiegmail.com). 
Thus, under certain conditions, the difference between $x(t)$ and $X^{T} \widehat{x}(t)$, where $\widehat{x}(t)$ is a solution of (1.1), converges to 0 as $t$ increases [3,5]. Indeed, differentiating the error $e(t):=x(t)-X^{T} \widehat{x}(t)$ and using (1.1) and (1.2), we obtain

$$
\begin{aligned}
\dot{e}(t) & =\dot{x}(t)-X^{T} \dot{\hat{x}}(t) \\
& =\widehat{H}^{T} x(t)+G^{T} \widehat{y}(t)+X^{T} B \widehat{u}(t)-X^{T}\left(A^{T} \widehat{x}(t)+B \widehat{u}(t)\right) \\
& =\widehat{H}^{T} e(t)-\Delta_{S}^{T} \widehat{x}(t),
\end{aligned}
$$

where $\Delta_{S}:=A X-X \widehat{H}-C G$. So, if the matrices $X, \widehat{H}$ and $G$ satisfy (1.3), then $\dot{e}(t)=\widehat{H}^{T} e(t)$, which gives

$$
e(t)=\exp \left(\widehat{H}^{T} t\right)\left(x_{0}-X^{T} \widehat{x}_{0}\right), t \geq 0 .
$$

Finally, requiring that the matrix $\widehat{H}$ be stable (all the eigenvalues of $\widehat{H}$ have negative realparts), it follows that the error $e(t)$ tends to 0 as $t$ increases. We point out that the construction of the stabilizing system (1.2) is a hard task since if the error matrix $\Delta_{S}$ is not exactly zero, then the error $e(t)$ may diverge even if $\widehat{H}$ is stable.

To solve (1.3), a first approach involves choosing the matrices $\widehat{H}$ and $G$ and then solving the Sylvester equation by a suitable method such as one of those described in $[1,5,10]$. However, generally these methods do not provide a well-conditioned solution $X$.

The matrix equation (1.3), where $A, C$ are given matrices and $H, X, G$ are to be found, is called a Sylvester-observer equation. In [7], Datta and Saad proposed an elegant method for the solution of equation (1.3) in the case where the system (1.1) is a Single-Input SingleOutput (SISO) system. By adapting this method and using the global Arnoldi process [15], a generalization of the Datta-Saad method for the case $\operatorname{rank}(C)=r$ has been proposed in [6]. To our knowledge, the methods described in $[6,7]$ are the only existing ones that handled the case of large matrices. These methods have in common that they exploit the resemblance between the Sylvester-observer equation and the Arnoldi recurrence stemming from the classical and global Arnoldi processes. Furthermore, these two methods transform by a particular assignment procedure an upper Hessenberg matrix into another one that has a well-chosen set of eigenvalues.

Throughout this paper, the symbols $\|\cdot\|_{2}$ and $\|\cdot\|_{F}$ denote the Euclidean norm and the Frobenius norm, respectively. The superscript ${ }^{T}$ denotes the transpose of a vector or a matrix. $0_{n \times r} \in \mathbb{R}^{n \times r}$ is the zero rectangular matrix with $n$ rows and $r$ columns, and $I_{r}, 0_{r} \in \mathbb{R}^{r \times r}$ are the identity matrix and zero matrix of dimension $r$, respectively. The spectrum of a square matrix $Z$ is denoted by $\sigma(Z)$.

In the present paper, we will use the block Arnoldi process and follow a similar approach to that used in $[6,7]$. Thus, letting $\mathbb{E}_{m}=\left[0_{r}, \ldots, 0_{r}, I_{r}\right]^{T} \in \mathbb{R}^{m r \times r}$ and taking $G=I_{q}$, $C=\widetilde{C} \mathbb{E}_{m}^{T} \in \mathbb{R}^{n \times m r}$, where $\widetilde{C} \in \mathbb{R}^{n \times r}$ is of full rank, $q=m r$, and $r \ll n$, the Sylvesterobserver equation (1.3) becomes

$$
A X-X \widehat{H}=\left[0_{n \times r}, \ldots, 0_{n \times r}, \widetilde{C}\right]=\widetilde{C} \mathbb{E}_{m}^{T},
$$

where the matrices $A$ and $\widetilde{C}$ are given, while $\widehat{H} \in \mathbb{R}^{m r \times m r}$ and $X \in \mathbb{R}^{n \times m r}$ are to be determined such that

- $\widehat{H}$ is a stable matrix, and

- the matrices $A$ and $\widehat{H}$ have a disjoint spectra, i.e., $\sigma(A) \cap \sigma(\widehat{H})=\emptyset$.

We mention that the condition $q=m r$ is not restrictive since in the case when $q=m r+r^{\prime}$ with $0<r^{\prime}<r$, we recover the case $q=m r$ by simply introducing the following dynamical 
system

$$
\begin{aligned}
& \dot{\widehat{x}}(t)=A^{T} \widehat{x}(t)+B \widehat{u}(t), \quad \widehat{x}(0)=\widehat{x}_{0}, \\
& \widehat{\widehat{y}}(t)=\bar{C}^{T} \widehat{x}(t), \quad t \geq 0,
\end{aligned}
$$

where $\bar{C}=\left[C, 0_{n \times\left(r-r^{\prime}\right)}\right] \in \mathbb{R}^{n \times q^{\prime}}, q^{\prime}=(m+1) r, \operatorname{rank}(\bar{C})=\operatorname{rank}(C)=r$, and $\overline{\widehat{y}}(t)=\left[\widehat{y}(t)^{T}, 0, \ldots, 0\right]^{T} \in \mathbb{R}^{(m+1) r}$. Indeed, it is easy to check that the dynamical systems (1.1) and (1.5) are equivalent.

We point out that for small problems, several works for solving Sylvester-observer equations have been published; see [3, 4, 20, 25]. Other existing numerical methods for small to medium problems include a parallel algorithm described in [2] and an SVD-based method proposed in [8]. However, the algorithms cited above cannot be applied to Sylvesterobserver equations whose coefficient matrix $A$ is very large. We also mention that the analysis given in [3] shows that the numerical stability of the Datta-Saad method can be significantly improved if the eigenvalues of the matrix $\widehat{H}$ are chosen well. We also point out that using the global Arnoldi process to solve the Sylvester-observer equation yields an upper Hessenberg matrix having exactly $m$ prescribed eigenvalues each of multiplicity $r$. This is not the case in the present work where the use of the block Arnoldi process allows certain control of the multiplicity of the desired values.

The paper is organized as follows. In Section 2, we give some background on the block Arnoldi process with some algebraic properties. Our proposed method for the Luenbergerobserver is developed in Section 3. More precisely, by using some matrix-valued polynomial properties in the first part of Section 3, we show how to choose the initial block Arnoldi matrix. Then in the second part of this section, we show how to replace the last block column of an upper block Hessenberg matrix such that the resulting matrix has a desired set of eigenvalues. We end Section 3 by showing how to construct the Luenberger-observer. A few numerical experiments are discussed in Section 4.

\section{The block Arnoldi process: background and notations.}

2.1. Definitions and notations. Let $A \in \mathbb{R}^{n \times n}, V \in \mathbb{R}^{n \times r}$ and $m>0$ be an integer. The $m$ th block Krylov space associated with the pair $(A, V)$ denoted by $\mathbb{K}_{m}(A, V)$ is the space of $\mathbb{R}^{n \times r}$ spanned by the columns of the matrices $V, A V, \ldots, A^{m-1} V$, i.e.,

$$
\mathbb{K}_{m}(A, V)=\operatorname{span}\left\{V, A V, \ldots, A^{m-1} V\right\} .
$$

Thus, this space is defined by

$$
\mathbb{K}_{m}(A, V)=\left\{\sum_{i=1}^{m} A^{i-1} V \Omega_{i}, \Omega_{i} \in \mathbb{R}^{r \times r}\right\},
$$

which can also be written as

$$
\mathbb{K}_{m}(A, V)=\left\{\mathcal{P}(A) \circ V, \mathcal{P} \in \mathbb{P}_{m-1, r}\right\},
$$

where $\mathbb{P}_{m, r}$ is the set of $r \times r$ matrix-valued polynomials of at most degree $m$, i.e., if $\psi_{i} \in \mathbb{R}^{r \times r}$ for $i=1, \ldots, m$, then

$$
\psi=\left(\psi_{i}\right) \in \mathbb{P}_{m, r} \Longleftrightarrow \psi(t)=\sum_{i=0}^{m} t^{i} \psi_{i},
$$


and the $\circ$ notation $[17,23]$ is defined as follows

$$
\psi(A) \circ V=\sum_{i=0}^{m} A^{i} V \psi_{i} .
$$

2.2. The block Arnoldi process. The block Arnoldi process can be seen as a natural generalization of the classical Arnoldi process [19]. When applied to the pair $(A, V)$, where $V \in \mathbb{R}^{n \times r}$, this process described by Algorithm 1 generates an orthonormal basis of the block Krylov subspace $\mathbb{K}_{m}(A, V)$.

Algorithm 1: The block Arnoldi algorithm.

Compute the $Q R$ decomposition of $V$, i.e., $\left[V_{1}, H_{1,0}\right]=Q R(V)$;

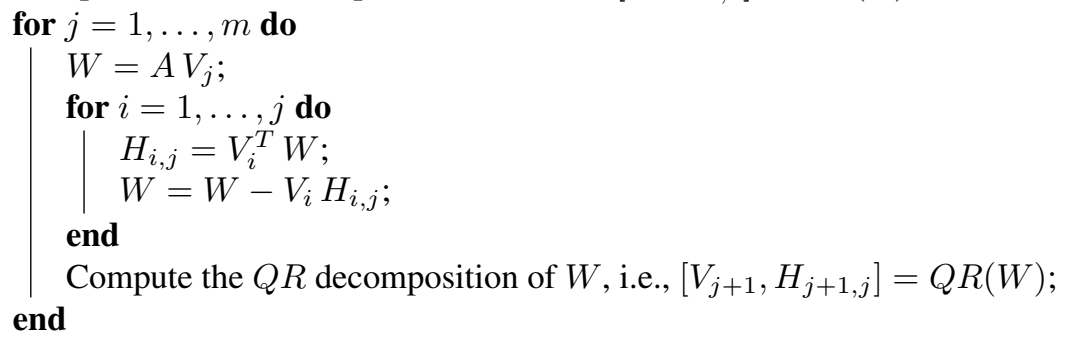

We assume that during the $m$ iterations of the above process, the upper triangular matrices $H_{j+1, j}$ are not rank deficient. In this case, Algorithm 1 constructs an orthonormal matrix $\mathbb{V}_{m}$ and an upper block Hessenberg matrix $\mathbb{H}_{m}$ that satisfy

$$
A \mathbb{V}_{m}=\mathbb{V}_{m} \mathbb{H}_{m}+V_{m+1} H_{m+1, m} \mathbb{E}_{m}^{T},
$$

where $\mathbb{V}_{m}=\left[V_{1}, V_{2}, \ldots, V_{m}\right] \in \mathbb{R}^{n \times m r}$, with $V_{i} \in \mathbb{R}^{n \times r}$ and $\mathbb{H}_{m}=\left(H_{i, j}\right) \in \mathbb{R}^{m r \times m r}$, with $H_{i, j} \in \mathbb{R}^{r \times r}$. The matrix $\mathbb{E}_{m}=\left[0_{r}, \ldots, 0_{r}, I_{r}\right]^{T}$ is the last $m r \times r$ block column of the identity matrix $I_{m r}$. We recall that if $k$ is the degree of the minimal polynomial of $V$ with respect to $A$, then there exists a matrix-valued polynomial $\mathcal{P}_{k}$ of degree $k$ such that $\mathcal{P}_{k}(A) \circ V_{1}=0_{n \times r}$. In this case, the block Krylov subspace $\mathbb{K}_{k}$ is invariant under $A$, which means that $\mathbb{K}_{k}=\mathbb{K}_{l}$ for $l \geq k$.

Notice that the orthonormal blocks $V_{i} \in \mathbb{R}^{n \times r}$ generated by the block Arnoldi process (Algorithm 1) can be expressed in terms of matrix-valued polynomials as

$$
V_{i+1}=\mathcal{P}_{i}(A) \circ V_{1}, \quad \text { for } i=0, \ldots, m,
$$

where $\mathcal{P}_{i}$ is an $r \times r$ matrix-valued polynomial of degree $i$. The following proposition, given in $[9,21]$, provides the key result that will allow us to use the block Arnoldi process for solving the Sylvester-observer equation (1.4).

PROPOSITION 2.1. Up to a multiplicative scalar $\rho_{i} \in \mathbb{R}$, the determinant of the matrixvalued polynomial $\mathcal{P}_{i}(t)$ given by (2.2) is equal to the characteristic polynomial of the block upper Hessenberg matrix $\mathbb{H}_{i}$, i.e.,

$$
\operatorname{det}\left(\mathcal{P}_{i}(t)\right)=\rho_{i} \operatorname{det}\left(\mathbb{H}_{i}-t I_{i r}\right) .
$$

3. The block Arnoldi process for the Sylvester-observer equation. As explained in the introduction, the main part of the Luenberger problem is to determine an $n \times m r$ matrix $X$ and an $m r \times m r$ matrix $\widehat{H}$ such that they are solutions of the Sylvester-observer equation

$$
A X-X \widehat{H}=\left[0_{n \times r}, \ldots, 0_{n \times r}, \widetilde{C}\right],
$$


and that $\sigma(\widehat{H})=\Gamma$, where $\Gamma:=\left\{\mu_{1}, \ldots, \mu_{m r}\right\}$ is a set of $m r$ suitably chosen scalars.

Taking advantage of the similarity between the previous Sylvester-observer equation and (2.1), we begin by looking for a starting matrix $Y$ such that the $(m+1)$ st matrix $V_{m+1}$ obtained by applying the block Arnoldi process to the pair $(A, Y)$ is equal to $\widetilde{\mathcal{C}}=\mathcal{P}(A) \circ Y$, which belongs to $\mathbb{K}_{m}(A, Y)$. However, this alone will not ensure that the spectrum of the obtained upper block Hessenberg matrix $\mathbb{H}_{m}$ is equal to $\Gamma$. This leads us to use a block assignment procedure in order to transform the matrix $\mathbb{H}_{m}$ into a new upper block Hessenberg matrix $\widehat{H}_{m}$ such that $\sigma\left(\widehat{H}_{m}\right)=\Gamma$.

3.1. Determining the initial block vector $V_{1}$. To determine $V_{1}$, we will use (2.2) and Proposition 2.1. We seek $Y \in \mathbb{R}^{n \times r}$ such that

$$
\mathcal{P}_{m}(A) \circ Y=\widetilde{\mathcal{C}},
$$

where $\operatorname{det}\left(\mathcal{P}_{m}(t)\right)=\prod_{i=1}^{m r}\left(t-\mu_{i}\right)$ is the characteristic polynomial of $\widehat{H}_{m}$. Once the block $Y$ is computed, we determine $V_{1}$ via the $\mathrm{QR}$ decomposition of $Y$, i.e., $\left[V_{1}, H_{1,0}\right]=\mathrm{QR}(Y)$.

Now, let $\widetilde{\mathcal{C}}=\left[\widetilde{c}_{1}, \widetilde{c}_{2}, \ldots, \widetilde{c}_{r}\right]$ with $\widetilde{c}_{i} \in \mathbb{R}^{n}$ and $\Lambda_{j+1}($ for $j=0, \ldots, m-1)$ is the $r \times r$ diagonal matrix given by

$$
\Lambda_{j+1}=\operatorname{diag}\left(\mu_{1+j r}, \mu_{2+j r}, \ldots, \mu_{r+j r}\right) .
$$

As the prescribed values $\mu_{1}, \ldots, \mu_{m r}$ are zeros of $\operatorname{det}\left(\mathcal{P}_{m}(t)\right)$, we propose to take

$$
\mathcal{P}_{m}(t)=\prod_{j=0}^{m-1} \phi_{j+1}(t) \quad \text { where } \quad \phi_{j+1}(t)=\left(t I_{r}-\Lambda_{j+1}\right) .
$$

It is clear that $\operatorname{det}\left(\mathcal{P}_{m}(t)\right)=\prod_{i=1}^{m r}\left(t-\mu_{i}\right)$.

Let $p_{m}^{(i)}(i=1, \ldots, r)$ be the scalar polynomial of degree $m$ defined by

$$
p_{m}^{(i)}(t)=\prod_{j=0}^{m-1}\left(t-\mu_{i+j r}\right) .
$$

Then we give the following result that will be useful later.

LEMMA 3.1. For every $Y \in \mathbb{R}^{n \times r}$, we have

$$
\mathcal{P}_{m}(A) \circ Y=\left[p_{m}^{(1)}(A) y_{1}, \ldots, p_{m}^{(r)}(A) y_{r}\right] .
$$

Proof. Using [11, Proposition 1], we can verify that

$$
\mathcal{P}_{m}(A) \circ Y=\left(\phi_{1} \phi_{2} \cdots \phi_{m}\right)(A) \circ Y=\left(\phi_{1} \phi_{2} \cdots \phi_{m-1}\right)(A) \circ\left(\phi_{m}(A) \circ Y\right) .
$$

Now, since $\phi_{m}(A) \circ Y=A Y-Y \Lambda_{m}=\left(\left(A-\mu_{1+(m-1) r} I_{n}\right) y_{1}, \ldots,\left(A-\mu_{m r} I_{n}\right) y_{r}\right)$, it follows that

$$
\begin{aligned}
\phi_{m-1}(A) \circ\left(\phi_{m}(A) \circ Y\right) & =A\left(\phi_{m}(A) \circ Y\right)-\left(\phi_{m}(A) \circ Y\right) \Lambda_{m-1} \\
& =\left[\prod_{j=m-2}^{m-1}\left(A-\mu_{1+j r} I_{n}\right) y_{1}, \ldots, \prod_{j=m-2}^{m-1}\left(A-\mu_{r+j r} I_{n}\right) y_{r}\right] .
\end{aligned}
$$


Thus, by induction and using (3.5), we obtain

$$
\begin{aligned}
\mathcal{P}_{m}(A) \circ Y & =\phi_{1}(A) \circ\left(\phi_{2}(A) \circ\left(\ldots \circ\left(\phi_{m}(A) \circ Y\right)\right)\right) \\
& =\left[\prod_{j=0}^{m-1}\left(A-\mu_{1+j r} I_{n}\right) y_{1}, \ldots, \prod_{j=0}^{m-1}\left(A-\mu_{r+j r} I_{n}\right) y_{r}\right] \\
& =\left[p_{m}^{(1)}(A) y_{1}, \ldots, p_{m}^{(r)}(A) y_{r}\right],
\end{aligned}
$$

which ends the proof.

Consequently, to obtain the solution $Y$ of (3.1), it suffices to compute the $r$ columns $y_{i}$ which satisfy

$$
p_{m}^{(i)}(A) y_{i}=\widetilde{c}_{i}
$$

To solve the $r$ systems (3.6), we proceed as in [7], and so we seek $y_{i}$ such that

$$
y_{i}=f^{(i)}(A) \widetilde{c}_{i} \quad \text { where } \quad f^{(i)}(t)=\frac{1}{p_{m}^{(i)}(t)}=\prod_{j=0}^{m-1} \frac{1}{\left(t-\mu_{i+j r}\right)} .
$$

As $Y=\left[y_{1}, \ldots, y_{r}\right]=\left[f^{(1)}(A) \widetilde{c}_{1}, \ldots, f^{(r)}(A) \widetilde{c}_{r}\right]$, referring to [5] and denoting by $\left[p_{m}^{(i)}\right]^{\prime}(t)$ the derivative of $p_{m}^{(i)}(t)$, we get for $i=1, \ldots, r$ the following expression

$$
f^{(i)}(t)=\frac{1}{p_{m}^{(i)}(t)}=\sum_{j=0}^{m-1} \frac{1}{\left[p_{m}^{(i)}\right]^{\prime}\left(\mu_{i+j r}\right) \times\left(t-\mu_{i+j r}\right)},
$$

where the derivatives $\left[p_{m}^{(i)}\right]^{\prime}\left(\mu_{i+j r}\right)$ are given by

$$
\left[p_{m}^{(i)}\right]^{\prime}\left(\mu_{i+j r}\right)=\prod_{\substack{k=0 \\ k \neq j}}^{m-1}\left(\mu_{i+j r}-\mu_{i+k r}\right)
$$

To guarantee that the fractions (3.7) are well defined, it is necessary to have

$$
\mu_{i+j r} \neq \mu_{i+k r} \quad \text { for } \quad j \neq k \quad \text { and } \quad j, k=0, \ldots, m-1 .
$$

Then, each $y_{i}$ is such that

$$
y_{i}=\sum_{j=0}^{m-1} \frac{1}{\left[p_{m}^{(i)}\right]^{\prime}\left(\mu_{i+j r}\right)} z_{j}^{(i)},
$$

where the $z_{j}^{(i)}$ are the solutions of the linear systems

$$
\left(A-\mu_{i+j r} I\right) z_{j}^{(i)}=\widetilde{c}_{i}, \quad \text { for } \quad i=1, \ldots, r \quad \text { and } \quad j=0, \ldots, m-1 .
$$

In order to solve these linear systems, we can apply independently a sparse direct method to each of the $m r$ shifted linear systems. In the present case, it may be preferable to use shifted Krylov subspace methods. Such methods allow the computation of approximate solutions for all the $m r$ shift values by generating one only projected subspace [12, 13, 22, 24, 26]. Here, 
we suggest to use $r$ times the restarted shifted Full Orthogonalization Method (rs-FOM) [22] to solve the $m$ shifted linear systems $\left(A-\mu_{i+j r} I\right) z_{j}^{(i)}=\widetilde{c}_{i}$ for $j=0, \ldots, m-1$. The index $i$ is to be fixed when solving the above shifted linear systems.

Before summarizing the restarted shifted FOM algorithm for solving (3.10), we provide an outline. The rs-FOM method described by Algorithm 2 makes use of the Arnoldi process [19] applied to the pair $(A, \widetilde{c})$ which constructs an orthonormal basis $\mathcal{V}_{k}=\left[v_{1}, \ldots, v_{k}\right] \in \mathbb{R}^{n \times k}$ of the Krylov subspace $K_{k}(A, \widetilde{c})=\operatorname{span}\left\{\widetilde{c}, A \widetilde{c}, \ldots, A^{k-1} \widetilde{c}\right\}$ and an upper Hessenberg matrix $\mathcal{H}_{k}=\left(h_{i, j}\right) \in \mathbb{R}^{k \times k}$. This method exploits the following two important properties; see, e.g., [7, 22]:

1. The shift invariance property of Krylov subspaces, i.e.,

$$
K_{k}(A, \widetilde{c})=K_{k}\left(A-\mu I_{n}, \widetilde{c}\right),
$$

and the classical and shifted Arnoldi relations

$$
\begin{aligned}
A \mathcal{V}_{k} & =\mathcal{V}_{k} \mathcal{H}_{k}+v_{k+1}\left[0, \ldots, h_{k+1, k}\right] \\
\left(A-\mu I_{n}\right) \mathcal{V}_{k} & =\mathcal{V}_{k}\left(\mathcal{H}_{k}-\mu I_{k}\right)+v_{k+1}\left[0, \ldots, h_{k+1, k}\right]
\end{aligned}
$$

2. The colinearity of the obtained residuals with the $(k+1)$ st basis vector $v_{k+1}$.

The restarted shifted $\operatorname{FOM}(k)$ algorithm is summarized as follows:

Algorithm 2: The restarted shifted $\operatorname{FOM}(k)$

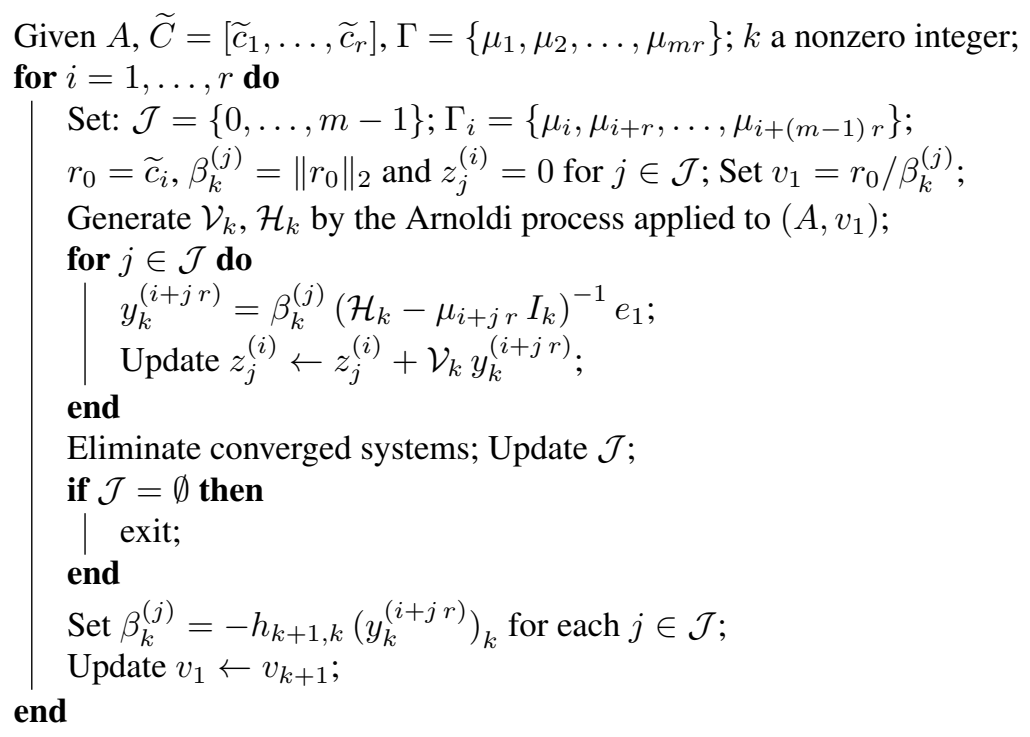

The shift invariance property (3.11) indicates that once a Krylov basis is generated for one of the linear systems (3.10), it can also be reused for all other linear systems. Thus, we do not need to construct an orthonormal basis for each shifted Krylov subspace $K_{k}\left(A-\mu I_{n}, \widetilde{c}\right)$. This strategy allows us to reduce the algorithmic cost considerably. For more details on the FOM and the restarted shifted FOM methods, we refer to [7, 19, 22, 26].

We mention that for $i=1, \ldots, r$, each column $y_{i}$ of the matrix $Y$ is simply computed via (3.9). Moreover, applying $m$ iterations of the block Arnoldi process to the pair $(A, Y)$ will produce an orthonormal matrix $\mathbb{V}_{m}$ and an upper block Hessenberg matrix that fulfill the 
Sylvester-observer equation

$$
A \mathbb{V}_{m}-\mathbb{V}_{m} \mathbb{H}_{m}=\left[0_{n \times r}, \ldots, 0_{n \times r}, V_{m+1} H_{m+1, m}\right] .
$$

3.2. Assigning eigenvalues to the block upper Hessenberg matrix $\mathbb{H}_{m}$. We begin this section by noticing that the block vector $V_{m+1}$ appearing in (3.12) is generally not colinear with $\widetilde{C}$, and thus, the eigenvalues of the matrix $\mathbb{H}_{m}$ do not necessarily coincide with the $m r$ chosen scalars $\left\{\mu_{k}\right\}_{k=1, \ldots, m r}$. Then, we have to transform $\mathbb{H}_{m}$ into a matrix $\widehat{H}_{m}$ so that the eigenvalues of the latter are exactly the $m r$ selected scalars $\left\{\mu_{k}\right\}_{k=1, \ldots, m r}$.

The particular case where $V_{m+1}$ and $\widetilde{C}$ are colinear, i.e., $\widetilde{C}=V_{m+1} \Psi_{m}$ (where $\Psi_{m}$ is some $r \times r$ matrix), can be considered as a "happy" case. In this case, we can easily verify that $\sigma\left(\mathbb{H}_{m}\right)=\left\{\mu_{k}\right\}_{k=1, \ldots, m r}=\Gamma$ thanks to (2.2) and Proposition 2.1.

To construct the matrix $\widehat{H}_{m}$, we need to define the following sequence of matrices $L_{i} \in \mathbb{R}^{m r \times r}:$

- Let $\mathbb{E}_{j}=\left[0_{r}, \ldots, 0_{r}, I_{r}, 0_{r}, \ldots, 0_{r}\right]^{T}$ be the $j$ th block column of the identity matrix.

- Let $L_{1} \in \mathbb{R}^{m r \times r}$ be such that

$$
L_{1}=\mathbb{E}_{1} H_{1,0}=\left[H_{1,0}^{T}, 0_{r}, \ldots, 0_{r}\right]^{T},
$$

where $H_{1,0}$ is the upper triangular matrix appearing in the QR decomposition of $Y$, i.e., $\left[V_{1}, H_{1,0}\right]=\mathrm{QR}(Y)$.

- For $i=1, \ldots, m$, let

$$
L_{i+1}=\mathbb{H}_{m} L_{i}-L_{i} \Lambda_{i} \in \mathbb{R}^{m r \times r},
$$

where $\Lambda_{i}$ is given by (3.2). We notice that $L_{i+1}^{(k)}$, the $k$ th column of $L_{i+1}$, is given by

$$
L_{i+1}^{(k)}=\left(\mathbb{H}_{m}-\mu_{k+(i-1) r} I_{m r}\right) L_{i}^{(k)} \quad \text { for } \quad k=1, \ldots, r .
$$

- Let $\boldsymbol{\alpha} \in \mathbb{R}^{r \times r}$ be defined as the product of the inverses of the $(m-1)$ block entries of the lower subdiagonal of the block upper Hessenberg matrix $\mathbb{H}_{m}$, i.e.,

$$
\boldsymbol{\alpha}=\prod_{i=1}^{m-1} H_{i+1, i}^{-1} .
$$

Notice that $H_{i+1, i}$ is invertible by assumption. Now, we give the following result that will be useful later.

Proposition 3.2. Let $S=L_{m+1}$. Then the matrix $S$ can be expressed in terms of a matrix-valued polynomial as

$$
S=\mathcal{P}_{m}\left(\mathbb{H}_{m}\right) \circ L_{1}
$$

Proof. By induction on $i=1, \ldots, m$ and using (3.15), it is easily seen that each column $S^{(k)}$ of the matrix $S$ can be expressed in terms of $\mathbb{H}_{m}$ and $L_{1}^{(k)}$ using the polynomial $p_{m}^{(k)}$, since

$$
\begin{aligned}
S^{(k)} & =L_{m+1}^{(k)}=\left[\left(\mathbb{H}_{m}-\mu_{k+(m-1) r} I_{m r}\right) \times \cdots \times\left(\mathbb{H}_{m}-\mu_{k} I_{m r}\right)\right] L_{1}^{(k)}, \\
& =\left[\prod_{j=0}^{m-1}\left(\mathbb{H}_{m}-\mu_{k+j r} I_{m r}\right)\right] L_{1}^{(k)}=p_{m}^{(k)}\left(\mathbb{H}_{m}\right) L_{1}^{(k)},
\end{aligned}
$$


where the last equality is justified by the definition of $p_{m}^{(k)}$ given in (3.3). Finally, using (3.4), we get the desired result.

We also have the following result, which gives the transformed matrix $\widehat{H}_{m}$ in terms of the upper block Hessenberg matrix $\mathbb{H}_{m}$.

PROPOSITION 3.3. The eigenvalues of the matrix $\widehat{H}_{m}=\mathbb{H}_{m}-S H_{1,0}^{-1} \boldsymbol{\alpha} \mathbb{E}_{m}^{T}$ are $\mu_{1}, \ldots, \mu_{m r}$.

Proof. Let $\mathbb{L}=\left[L_{1}, L_{2}, \ldots, L_{m}\right] \in \mathbb{R}^{m r \times m r}$, where $L_{i}$ is defined by (3.14), and let $B$ be the bi-diagonal block matrix defined as follows

$$
B=\left[\begin{array}{ccccc}
\Lambda_{1} & 0 & & \cdots & 0 \\
I_{r} & \Lambda_{2} & \ddots & & \\
0 & \ddots & \ddots & \ddots & \vdots \\
\vdots & \ddots & \ddots & \ddots & 0 \\
0 & \cdots & 0 & I_{r} & \Lambda_{m}
\end{array}\right] .
$$

It is easy to verify that the matrix $\mathbb{L}$ is an upper triangular matrix whose $k$ th diagonal block entry $\mathbb{L}_{k, k}(k=1, \ldots, m)$ is given by

$$
\mathbb{L}_{k, k}=H_{k, k-1} \times \cdots \times H_{2,1} \times H_{1,0}=\prod_{i=k}^{1} H_{i, i-1} .
$$

We recall that the matrices $H_{i, i-1}$ are upper triangular matrices computed by a QR decomposition. These matrices are assumed to be nonsingular. Therefore the matrix $\mathbb{L}$ is also nonsingular. In addition, by using (3.14), we have

$$
\begin{aligned}
\mathbb{H}_{m} \mathbb{L}-\mathbb{L} B= & {\left[\mathbb{H}_{m} L_{1}, \ldots, \mathbb{H}_{m} L_{m-1}, \mathbb{H}_{m} L_{m}\right] } \\
& -\left[L_{1} \Lambda_{1}+L_{2}, \ldots, L_{m-1} \Lambda_{m-1}+L_{m}, L_{m} \Lambda_{m}\right] \\
= & {\left[\mathbb{H}_{m} L_{1}-L_{1} \Lambda_{1}-L_{2}, \ldots, \mathbb{H}_{m} L_{m-1}-L_{m-1} \Lambda_{m-1}-L_{m},\right.} \\
& \left.\mathbb{H}_{m} L_{m}-L_{m} \Lambda_{m}\right] \\
= & {\left[0_{m r \times r}, \ldots, 0_{m r \times r}, S\right] . }
\end{aligned}
$$

Multiplying from the right by $\mathbb{L}^{-1}$, we get

$$
\mathbb{H}_{m}-\left[0_{m r \times r}, \ldots, 0_{m r \times r}, S\right] \mathbb{L}^{-1}=\mathbb{L} B \mathbb{L}^{-1} .
$$

But $\left[0_{m r \times r}, \ldots, 0_{m r \times r}, S\right] \mathbb{L}^{-1}=\left[0_{m r \times r}, \ldots, 0_{m r \times r}, S \mathbb{L}_{m, m}^{-1}\right]=S H_{1,0}^{-1} \boldsymbol{\alpha} \mathbb{E}_{m}^{T}$.

Finally, we get

$$
\widehat{H}_{m}=\mathbb{H}_{m}-S H_{1,0}^{-1} \boldsymbol{\alpha} \mathbb{E}_{m}^{T}=\mathbb{L} B \mathbb{L}^{-1},
$$

which shows that $\widehat{H}_{m}$ and $B$ are similar and have the same eigenvalues $\mu_{1}, \ldots, \mu_{m r}$.

In the following lemmas, we state some useful results to be used later.

LEMMA 3.4. Let $\mathbb{V}_{m}$ and $\mathbb{H}_{m}$ be the matrices generated after $m$ iterations of the block Arnoldi process applied to the pair $(A, Y)$ and let $\mathcal{P}$ be a matrix-valued polynomial of degree $j<m$. Then

$$
\mathbb{V}_{m}^{T}\left(\mathcal{P}(A) \circ V_{1}\right)=\mathcal{P}\left(\mathbb{H}_{m}\right) \circ \mathbb{E}_{1}
$$

and

$$
\mathbb{V}_{m}^{T}(\mathcal{P}(A) \circ Y)=\mathcal{P}\left(\mathbb{H}_{m}\right) \circ L_{1},
$$

where $L_{1}$ is defined by (3.13). 
Proof. Using (2.1), we can show by induction that for $j=1, \ldots, m-1$, we have

$$
A^{j} V_{1}=\mathbb{V}_{m} \mathbb{H}_{m}^{j} \mathbb{E}_{1}
$$

So, for every matrix-valued polynomial $\mathcal{P}$ of degree $j<m$ such that $\mathcal{P}(t)=\sum_{i=0}^{j} t^{i} \Omega_{i}$, where $\Omega_{i} \in \mathbb{R}^{r \times r}$, we have

$$
\mathcal{P}(A) \circ V_{1}=\sum_{i=0}^{j} A^{i} V_{1} \Omega_{i}=\sum_{i=0}^{j} \mathbb{V}_{m} \mathbb{H}_{m}^{i} \mathbb{E}_{1} \Omega_{i}=\mathbb{V}_{m}\left(\mathcal{P}\left(\mathbb{H}_{m}\right) \circ \mathbb{E}_{1}\right) .
$$

Finally, since the matrix $\mathbb{V}_{m}$ is orthonormal, we have $\mathbb{V}_{m}^{T}\left(\mathcal{P}(A) \circ V_{1}\right)=\mathcal{P}\left(\mathbb{H}_{m}\right) \circ \mathbb{E}_{1}$, which proves (3.18).

The second equality (3.19) can be proved as follows. Using the fact that $Y=V_{1} H_{1,0}$, $L_{1}=\mathbb{E}_{1} H_{1,0}$, and the relation (3.20), we get

$$
\begin{aligned}
\mathbb{V}_{m}^{T}(\mathcal{P}(A) \circ Y)= & \mathbb{V}_{m}^{T}\left(\sum_{i=0}^{j} A^{i} V_{1} H_{1,0} \Omega_{i}\right)=\mathbb{V}_{m}^{T}\left(\sum_{i=0}^{j} \mathbb{V}_{m} \mathbb{H}_{m}^{i} \mathbb{E}_{1} H_{1,0} \Omega_{i}\right) \\
& =\sum_{i=0}^{j} \mathbb{H}_{m}^{i} L_{1} \Omega_{i}=\mathcal{P}\left(\mathbb{H}_{m}\right) \circ L_{1},
\end{aligned}
$$

which completes the proof.

LEMMA 3.5. Let $\mathbb{H}_{m+1}=\left(H_{i, j}\right) \in \mathbb{R}^{(m+1) r \times(m+1) r}$ be an upper block Hessenberg matrix with $H_{i, j} \in \mathbb{R}^{r \times r}$ and $\mathcal{P}$ a monic matrix-valued polynomial of degree $m$. Then

$$
\mathbb{E}_{m+1}^{T}\left(\mathcal{P}\left(\mathbb{H}_{m+1}\right) \circ \mathbb{E}_{1}\right)=H_{m+1, m} \times \cdots \times H_{2,1} .
$$

Proof. To prove (3.21), we have to compute the products $\mathbb{E}_{m+1}^{T} \mathbb{H}_{m+1}^{i} \mathbb{E}_{1}, i=1, \ldots, m$. Notice that $\mathbb{E}_{m+1}^{T} \mathbb{H}_{m+1}^{i} \mathbb{E}_{1}$ is the $(i+1)$ st entry of the first block column of $\mathbb{H}_{m+1}^{i}$, and as $\mathbb{H}_{m+1}$ is an upper block Hessenberg matrix, we can show that

$$
\mathbb{E}_{m+1}^{T} \mathbb{H}_{m+1}^{i} \mathbb{E}_{1}=0_{r} \quad \text { for } \quad i=0,1, \ldots, m-1,
$$

and for $i=m$, we have

$$
\mathbb{E}_{m+1}^{T} \mathbb{H}_{m+1}^{m} \mathbb{E}_{1}=H_{m+1, m} \times \cdots \times H_{2,1} .
$$

Finally, from the above equalities, we deduce that for every monic matrix-valued polynomial $\mathcal{P}$ of degree $m$, we have

$$
E_{m+1}^{T} \mathcal{P}\left(\mathbb{H}_{m+1}\right) \circ \mathbb{E}_{1}=H_{m+1, m} \times \cdots \times H_{2,1},
$$

which completes the proof.

In the following result, we give a new expression for the matrix $\widehat{H}_{m}$, which is, from a practical and computational point of view, more convenient than the first expression given in Proposition 3.3.

PROPOSITION 3.6. Let $\mathbb{V}_{m+1}=\left[\mathbb{V}_{m}, V_{m+1}\right]$, and let $\mathbb{H}_{m}$ be the orthonormal and the upper block Hessenberg matrices constructed by the block Arnoldi process at step m. Let also

$$
\boldsymbol{\beta}_{m}=\left(V_{m+1}^{T} \widetilde{\mathcal{C}}\right)^{-1} H_{m+1, m} .
$$


Then the matrix $\widehat{H}_{m}$ can be written as

$$
\widehat{H}_{m}=\mathbb{H}_{m}-F \mathbb{E}_{m}^{T}
$$

where $F:=\mathbb{V}_{m}^{T} \widetilde{C} \boldsymbol{\beta}_{m}$. Moreover, the matrix $\widehat{H}_{m}$ satisfies the Arnoldi-like relation

$$
A \mathbb{V}_{m}-\mathbb{V}_{m} \widehat{H}_{m}=\widetilde{\mathcal{C}} \boldsymbol{\beta}_{m} \mathbb{E}_{m}^{T} .
$$

Proof. To prove (3.24), we have to show that $F=\mathbb{V}_{m}^{T} \widetilde{C} \boldsymbol{\beta}_{m}$ also can be written as

$$
F=S H_{1,0}^{-1} \boldsymbol{\alpha} .
$$

To verify the above equality, we first notice that the matrix-valued polynomial $\mathcal{P}_{m}$ can be expressed as

$$
\mathcal{P}_{m}(t)=\left(t I_{r}-\Lambda_{m}\right) \mathcal{P}_{m-1}(t)=Q(t) \mathcal{P}_{m-1}(t)
$$

Therefore,

$$
\begin{aligned}
\mathcal{P}_{m}(A) \circ Y & =\left(Q \mathcal{P}_{m-1}\right)(A) \circ Y \\
& =Q(A) \circ\left(\mathcal{P}_{m-1}(A) \circ Y\right) \\
& =Q(A) \circ\left(\mathbb{V}_{m}\left(\mathcal{P}_{m-1}\left(\mathbb{H}_{m}\right) \circ L_{1}\right)\right) \\
& =A \mathbb{V}_{m}\left(\mathcal{P}_{m-1}\left(\mathbb{H}_{m}\right) \circ L_{1}\right)-\left(\mathbb{V}_{m}\left(\mathcal{P}_{m-1}\left(\mathbb{H}_{m}\right) \circ L_{1}\right)\right) \Lambda_{m}
\end{aligned}
$$

Multiplying the last relation from the left by $\mathbb{V}_{m}^{T}$, we get

$$
\begin{aligned}
\mathbb{V}_{m}^{T}\left(\mathcal{P}_{m}(A) \circ Y\right) & =\mathbb{H}_{m}\left(\mathcal{P}_{m-1}\left(\mathbb{H}_{m}\right) \circ L_{1}\right)-\left(\mathcal{P}_{m-1}\left(\mathbb{H}_{m}\right) \circ L_{1}\right) \Lambda_{m} \\
& =Q\left(\mathbb{H}_{m}\right) \circ\left(\mathcal{P}_{m-1}\left(\mathbb{H}_{m}\right) \circ L_{1}\right) \\
& =\mathcal{P}_{m}\left(\mathbb{H}_{m}\right) \circ L_{1} .
\end{aligned}
$$

Now, using (3.17), it follows that

$$
F=\mathbb{V}_{m}^{T} \widetilde{\mathcal{C}} \boldsymbol{\beta}_{m}=\mathbb{V}_{m}^{T}\left(\mathcal{P}_{m}(A) \circ Y\right) \boldsymbol{\beta}_{m}=\left(\mathcal{P}_{m}\left(\mathbb{H}_{m}\right) \circ L_{1}\right) \boldsymbol{\beta}_{m}=S \boldsymbol{\beta}_{m} .
$$

Due to the orthogonality of $\left\{V_{1}, \ldots, V_{m+1}\right\}$ and to (3.20), we obtain

$$
\begin{aligned}
\boldsymbol{\beta}_{m} & =\left(V_{m+1}^{T} \widetilde{\mathcal{C}}\right)^{-1} H_{m+1, m} \\
& =\left(V_{m+1}^{T}\left(\mathcal{P}_{m}(A) \circ Y\right)\right)^{-1} H_{m+1, m} \\
& =\left(\sum_{i=0}^{m} V_{m+1}^{T} A^{i} V_{1} H_{1,0} \Omega_{i}\right)^{-1} H_{m+1, m}\left(\text { where } \Omega_{i} \in \mathbb{R}^{r \times r} \text { and } \Omega_{m}=I_{r}\right) \\
& =\left(V_{m+1}^{T} A^{m} V_{1} H_{1,0} \Omega_{m}\right)^{-1} H_{m+1, m} \\
& =\left(V_{m+1}^{T} \mathbb{V}_{m+1} \mathbb{H}_{m+1}^{m} \mathbb{E}_{1} H_{1,0}\right)^{-1} H_{m+1, m} \\
& =H_{1,0}^{-1}\left(\mathbb{E}_{m+1}^{T} \mathbb{H}_{m+1}^{m} \mathbb{E}_{1}\right)^{-1} H_{m+1, m} \\
& =H_{1,0}^{-1} \boldsymbol{\alpha},
\end{aligned}
$$

where, in the last equality, we used (3.22) and (3.16). Thus, we have a new expression for $\boldsymbol{\beta}_{m}$,

$$
\boldsymbol{\beta}_{m}=\prod_{i=0}^{m-1} H_{i+1, i}^{-1}
$$


and we get the desired result (3.26). Finally, since $\widehat{H}_{m}=\mathbb{H}_{m}-S H_{1,0}^{-1} \boldsymbol{\alpha} \mathbb{E}_{m}^{T}$, the relation (3.24) follows immediately.

To prove (3.25), we notice that since, $\widetilde{\mathcal{C}}=\mathcal{P}_{m}(A) \circ Y$, then $\widetilde{\mathcal{C}} \in \mathbb{K}_{m+1}\left(A, V_{1}\right)$. Therefore there exists $\bar{\Omega}_{m} \in \mathbb{R}^{m r \times r}$ and $\bar{g} \in \mathbb{R}^{r \times r}$ such that

$$
\widetilde{\mathcal{C}}=\mathbb{V}_{m} \bar{\Omega}_{m}+V_{m+1} \bar{g}
$$

Using the fact that $\mathbb{V}_{m+1}$ is orthonormal, we obtain

$$
\bar{\Omega}_{m}=\mathbb{V}_{m}^{T} \widetilde{\mathcal{C}} \text { and } \bar{g}=V_{m+1}^{T} \widetilde{\mathcal{C}} .
$$

Therefore,

$$
\begin{aligned}
\widetilde{\mathcal{C}} \boldsymbol{\beta}_{m} & =\mathbb{V}_{m} \bar{\Omega}_{m} \boldsymbol{\beta}_{m}+V_{m+1} \bar{g} \boldsymbol{\beta}_{m} \\
& =\mathbb{V}_{m} \mathbb{V}_{m}^{T} \widetilde{\mathcal{C}} \boldsymbol{\beta}_{m}+V_{m+1} V_{m+1}^{T} \widetilde{\mathcal{C}} \boldsymbol{\beta}_{m} \\
& =\mathbb{V}_{m} F+V_{m+1} H_{m+1, m},
\end{aligned}
$$

where $F=\mathbb{V}_{m}^{T} \widetilde{\mathcal{C}} \boldsymbol{\beta}_{m}$. Multiplying $\widetilde{\mathcal{C}} \boldsymbol{\beta}_{m}$ from the right by $\mathbb{E}_{m}^{T}$, we get

$$
\widetilde{\mathcal{C}} \boldsymbol{\beta}_{m} \mathbb{E}_{m}^{T}=\mathbb{V}_{m} F \mathbb{E}_{m}^{T}+V_{m+1} H_{m+1, m} \mathbb{E}_{m}^{T} .
$$

Invoking (2.1), we obtain the Arnoldi-like relation (3.25),

$$
A \mathbb{V}_{m}-\mathbb{V}_{m} \mathbb{H}_{m}+\mathbb{V}_{m} F \mathbb{E}_{m}^{T}=\widetilde{\mathcal{C}} \boldsymbol{\beta}_{m} \mathbb{E}_{m}^{T}
$$

To recover the Sylvester-observer form (1.4), we express the matrix equation (3.25) as

$$
A \mathbb{V}_{m}-\mathbb{V}_{m} \widehat{H}_{m}=\left[0_{n \times r}, \ldots, 0_{n \times r}, \widetilde{\mathcal{C}} \boldsymbol{\beta}_{m}\right]
$$

and define $D$ as the last block-column of the matrix $A \mathbb{V}_{m}-\mathbb{V}_{m} \widehat{H}_{m}$, i.e., $D=\widetilde{\mathcal{C}} \boldsymbol{\beta}_{m}$. Let $\widetilde{\mathcal{C}}^{+}=\left(\widetilde{\mathcal{C}} \widetilde{\mathcal{C}}^{T}\right)^{-1} \widetilde{\mathcal{C}}^{T}$ be the pseudo-inverse of $\widetilde{\mathcal{C}}$. Then we can write $\boldsymbol{\beta}_{m}=\widetilde{\mathcal{C}}^{+} D$. Now, introducing the following block diagonal matrix

$$
\Theta=\left[\begin{array}{cccc}
I_{r} & 0_{r} & \cdots & 0_{r} \\
0_{r} & \ddots & \ddots & \vdots \\
\vdots & \ddots & I_{r} & 0_{r} \\
0_{r} & \cdots & 0_{r} & \boldsymbol{\beta}_{m}^{-1}
\end{array}\right],
$$

we rewrite (3.28) as follows

$$
A \mathbb{V}_{m} \Theta-\mathbb{V}_{m} \Theta \Theta^{-1} \widehat{H}_{m} \Theta=\left[0_{n \times r}, \ldots, 0_{n \times r}, \widetilde{\mathcal{C}}\right]
$$

and finally take

$$
X=\mathbb{V}_{m} \Theta \text {, and } \widehat{H}=\Theta^{-1} \widehat{H}_{m} \Theta .
$$

The block Arnoldi algorithm for solving the the multi-output Sylvester-observer problem is summarized in Algorithm 3. 
Algorithm 3: The block Arnoldi algorithm for multi-output Sylvester-observer equation.

Input: $A \in \mathbb{R}^{n \times n}, \widetilde{C}=\left(\widetilde{c}_{1}, \ldots, \widetilde{c}_{r}\right) \in \mathbb{R}^{n \times r}$ with $\operatorname{rank}(\widetilde{C})=r$ and $\Gamma=\left\{\mu_{1}, \mu_{2}, \ldots, \mu_{m r}\right\}$ satisfying (3.8).

1. Solve the linear problem $\mathcal{P}_{m}(A) \circ Y=\widetilde{\mathcal{C}}$, i.e.,

(a) Solve the $m r$ linear systems

$$
\left(A-\mu_{i+j r} I_{m r}\right) z_{j}^{(i)}=\widetilde{c}_{i}
$$

for $i=1, \ldots, r$ and $j=0, \ldots, m-1$.

(b) For $i=1, \ldots, r$, compute

$$
y_{i}=\sum_{j=0}^{m-1} \gamma_{j} z_{j}^{(i)} \quad \text { where } \quad \gamma_{j}=\left(\prod_{\substack{k=0 \\ k \neq j}}^{m-1}\left(\mu_{i+j r}-\mu_{i+k r}\right)\right)^{-1} .
$$

2. Define $Y=\left[y_{1}, \ldots, y_{r}\right]$ and apply Algorithm 1 to the pair $(A, Y)$ to get

$$
\mathbb{V}_{m+1}=\left[V_{1}, \ldots, V_{m}, V_{m+1}\right] \text { and } \mathbb{H}_{m}=\left[H_{i, j}\right] .
$$

3. Modify $\mathbb{H}_{m}$ to get $\widehat{H}$ such that $\sigma(\widehat{H})=\left\{\mu_{1}, \mu_{2}, \ldots, \mu_{m r}\right\}$, i.e.,

(a) Compute $\boldsymbol{\beta}_{m}=\left(V_{m+1}^{T} \widetilde{\mathcal{C}}\right)^{-1}, H_{m+1, m}=\prod_{i=0}^{m-1} H_{i+1, i}^{-1}$ and

$$
F=\mathbb{V}_{m}^{T} \widetilde{\mathcal{C}} \boldsymbol{\beta}_{m}
$$

(b) Define $\widehat{H}_{m}=\mathbb{H}_{m}-F \mathbb{E}_{m}^{T}$.

(c) Compute $\Theta$ using (3.29).

4. Take $X=\mathbb{V}_{m} \Theta$ and $\widehat{H}=\Theta^{-1} \widehat{H}_{m} \Theta$.

REMARK 3.7. Before ending this section, we make a few remarks:

1. It should be noted from (3.8) that the parameter $\gamma_{j}$ in step 1(b) of Algorithm 3 is well defined. Moreover, we mention that this condition is the only constraint which must be satisfied by the scalars $\mu_{i}$. Thus these values are not inevitably all distinct.

2. The numerical tests we have performed show that computing $\boldsymbol{\beta}_{m}$ in step 3(a) by formula (3.27) gives better results than by using the formula (3.23). In this case, it is more convenient to write (3.27) as follows

$$
\boldsymbol{\beta}_{m}=\left(\prod_{i=0}^{m-1} H_{m-i, m-i-1}\right)^{-1}=\left(\prod_{i=m-1}^{0} H_{i+1, i}\right)^{-1} .
$$

3. From (3.29), we see that $\Theta$ is nearly equal to the identity matrix apart from the last $r \times r$ block. Thus, the solutions $X$ and $\widehat{H}$ given by (3.30) are not expensive to compute. Moreover, since $\mathbb{V}_{m}$ is orthonormal,

$$
\|X\|_{F}=\|\Theta\|_{F}=\left\|\boldsymbol{\beta}_{m}^{-1}\right\|_{F} .
$$

4. Since $m, r \ll n$, the algorithmic cost of Algorithm 3 is generally dominated by the solution of the $m r$ shifted linear systems.

4. Numerical experiments. The numerical tests described in this section were carried out on an Intel Core i5 workstation using MATLAB (version R2015a) and floating point 


\section{ETNA}

Kent State University and

Johann Radon Institute (RICAM)

BLOCK ARNOLDI METHOD FOR SYLVESTER-OBSERVER EQUATION
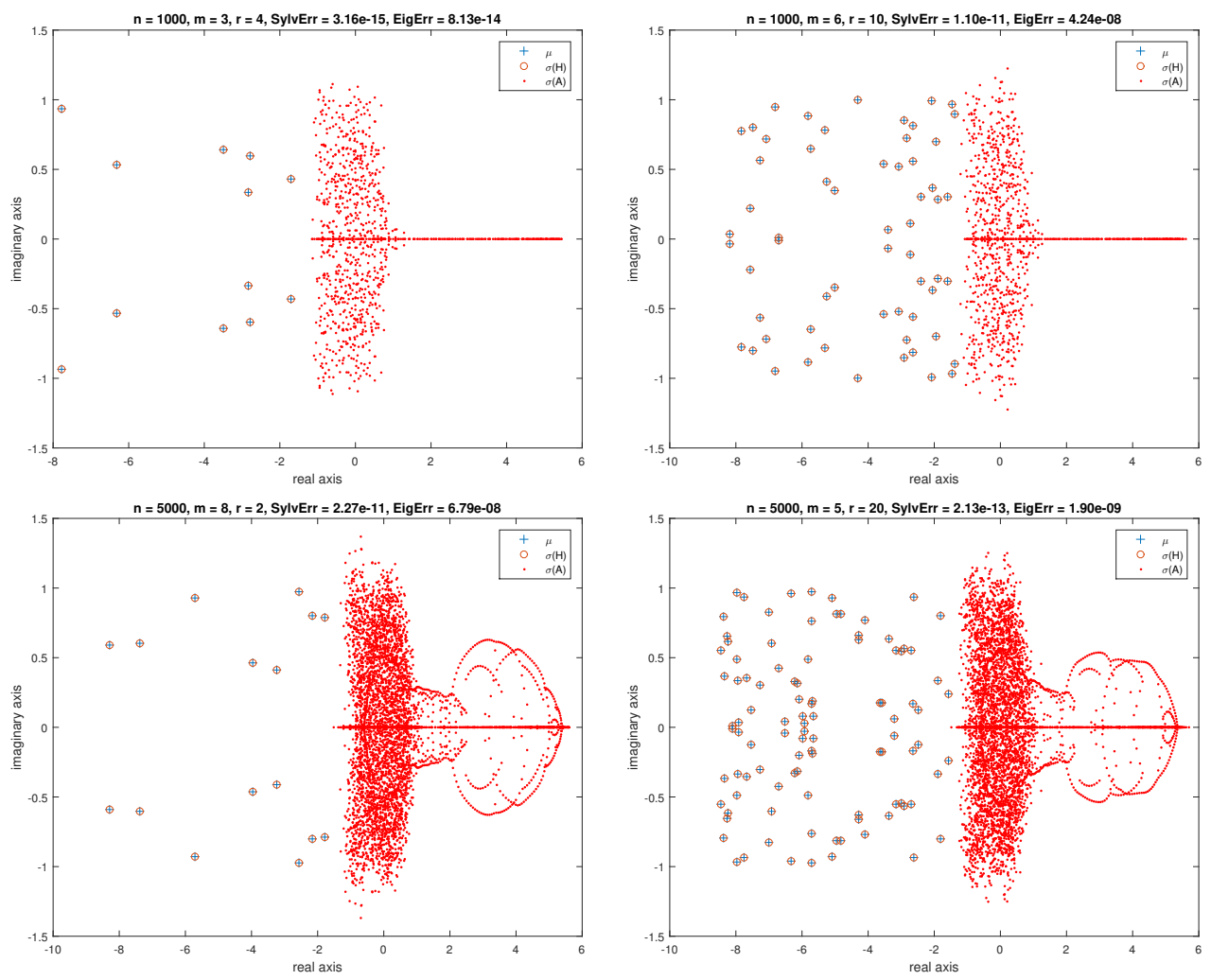

FIG. 4.1. Figures for Example 4.1. Eigenvalues of $A(\cdot)$, Eigenvalues of $\widehat{H}(\circ)$, Scalars $\left\{\mu_{k}\right\}_{k=1, \ldots, m r}(+)$. Top left: plot obtained with $n=1000, m=3, r=4$. Top right: plot obtained with $n=1000, m=6, r=10$. Bottom left: plot obtained with $n=5000, m=8, r=2$. Bottom right: plot obtained with $n=5000, m=5$, $r=20$.

arithmetic with 16 significant decimal digits. For solving the shifted linear systems, we used Gaussian elimination or the restarted shifted $\operatorname{FOM}(k)$ method (Algorithm 2) with $k=50$ and a maximum of rest $_{\max }=50$ restarts. We considered that a shifted system converged if the norm of the corresponding residual is less or equal to $t o l=10^{-10}$. The $n \times r$ block $\widetilde{\mathcal{C}}$ is generated randomly via the MATLAB function $\mathcal{\mathcal { C }}=\operatorname{rand}(n, r)$.

In all the tables, except for the last one, we reported the relative error norms for the Sylvester-observer equation given by

$$
\text { SylvErr }=\left\|\left(A_{1} X-X \widehat{H}\right)-\widetilde{\mathcal{C}} \mathbb{E}_{m}^{T}\right\|_{2} /\|\widetilde{\mathcal{C}}\|_{2}
$$

and the relative distance between the eigenvalues of $\widehat{H}$ and the values of $\mu$ given by

$$
\text { EigErr }=\|\sigma(\widehat{H})-\Gamma\|_{2} /\|\Gamma\|_{2} .
$$

EXAMPLE 4.1. In this first example, the test matrix $A=A_{1}$ is generated randomly by the MATLAB command $A \_l=\operatorname{sparse}(B-\operatorname{tril}(B,-5)-\operatorname{triu}(B, 7))$, where $B=\operatorname{rand}(n)$. The set of prescribed values is $\Gamma_{1}=\left\{z_{k}, \bar{z}_{k}\right\}$, where $\operatorname{Re}\left(z_{-} k\right)=-7 * \operatorname{rand}+\min (\operatorname{real}(\operatorname{eig}(A)))$ and $\operatorname{Im}\left(z_{-} k\right)=$ rand for $k=1, \ldots, m r$.

In Figure 4.1, we plotted the eigenvalues $\sigma\left(A_{1}\right)$ of the matrix $A_{1}$ which are marked by dots and the eigenvalues $\sigma(\widehat{H})$ of the matrix $\widehat{H}$ which are marked by circles. The prescribed 


\section{ETNA}

Kent State University and

Johann Radon Institute (RICAM)

TABLE 4.1

Results for Example 4.1 with $A=A_{1}$. The prescribed set of eigenvalues is $\Gamma=\Gamma_{1}$. Here, we used Gaussian elimination to solve the linear systems.

\begin{tabular}{ccccc}
$n$ & $m$ & $r$ & SylvErr & EigErr \\
\hline 1000 & 3 & 4 & $3.16 \times 10^{-15}$ & $8.13 \times 10^{-14}$ \\
5000 & 8 & 2 & $2.27 \times 10^{-11}$ & $6.79 \times 10^{-08}$ \\
1000 & 6 & 10 & $1.10 \times 10^{-11}$ & $4.28 \times 10^{-10}$ \\
5000 & 5 & 20 & $2.13 \times 10^{-13}$ & $1.90 \times 10^{-09}$
\end{tabular}

values $\mu_{1}, \ldots, \mu_{m r}$ are marked by plus signs. As can be seen from this figure and Table 4.1, the obtained results are quite good.

EXAMPLE 4.2. For the second example, we consider two matrices: $A_{2}=$ (poisson,100), where $n=10000, n n z\left(A_{2}\right)=49600$ and $A_{3}=($ wathen,70,100), where $n=21341$ and $n n z\left(A_{3}\right)=330361$. These matrices come from a set of test matrices collected by Higham [14] and can be generated via the MATLAB function gallery. As these matrices are symmetric and positive definite, we consider a set $\Gamma=\left\{\mu_{1}, \ldots, \mu_{m r}\right\}$ of $m r$ distinct negative real values. To show the influence of the prescribed set of eigenvalues $\Gamma$, we consider three different choices of $\Gamma$. More precisely, the considered sets are generated randomly by the MATLAB function rand via Gamma $=-c * \operatorname{rand}(m * r, 1)$, where $c$ is a positive integer. The obtained results for different values of $c, m$, and $r$ are reported in Table 4.2a, Table 4.2b, and Table 4.2c. Again, in these tables we report the relative error norms for the Sylvester-observer equation and between the eigenvalues of $\widehat{H}$ and the values of $\mu$. We also report the conditioning number of the approximation $X$.

As observed from the results of these tables, the accuracy is quite good even for small $\mathrm{m}$. We notice that as the matrices are sparse and structured, we can also use Gaussian elimination for solving the shifted linear systems. In this case, and as shown in Table $4.2 \mathrm{c}$, the results obtained are better than those from the restarted shifted FOM method.

EXAMPLE 4.3. In this example, we considered the matrix given by Datta and Saad [7]. Let $L=-\operatorname{diag}\left(l_{1}, \ldots, l_{p}\right)$ and $D=\operatorname{diag}\left(d_{1}, \ldots, d_{p}\right)$, two diagonal matrices of size $p \times p$. Then

$$
A=A_{4}=\left[\begin{array}{cc}
0_{p} & I_{p} \\
L & D
\end{array}\right]
$$

We mention that the eigenvalues of $A_{4}$ are the solutions of the quadratic equations

$$
x^{2}-d_{k} x-l_{k}=0, \quad k=1, \ldots, p .
$$

Thus, taking $d_{k}=2 \alpha_{k}$ and $l_{k}=-\left(\alpha_{k}^{2}+\beta_{k}^{2}\right)$ gives that $\sigma\left(A_{4}\right)=\left\{\lambda_{k}, \bar{\lambda}_{k}\right\}_{k=1, \ldots, p}$, where $\lambda_{k}=\alpha_{k}+\imath \beta_{k}$. In this experiment, the parameters $\alpha_{k}, \beta_{k}$ were random values uniformly distributed in $[-1,1]$. To choose $\Gamma$, the set of desired eigenvalues, we adopt the strategy described in [3]. More precisely, we choose the $\mu_{k}$ to be zeros of a Chebyshev polynomial for the interval $[a+\imath b, a-\imath b]$, where $a=-1+\min _{d \in \sigma\left(A_{4}\right)} \Re(d)$ and $b=\max _{d \in \sigma\left(A_{4}\right)} \Im(d)$.

Our experiments were run with $n=2 p=20000$ and we used different values of $m$ and $r$. We also point out that as the matrix $A_{4}$ is sparse and structured, we used Gaussian elimination to solve the shifted linear systems. The results displayed in Table 4.3a are those obtained with $m r$ distinct values $\mu_{1}, \ldots, \mu_{m r}$ invariant under complex conjugation. These parameters $\mu_{k}$ are zeros of the Chebyshev polynomial of the first kind of degree $m r$ for the interval $[a+\imath b, a-\imath b]$. To compare with the global Arnoldi algorithm for multiple-output Sylvester-observer equation [6], we give in Table $4.3 \mathrm{~b}$ and Table $4.3 \mathrm{c}$ the results obtained with 


\section{ETNA}

Kent State University and

Johann Radon Institute (RICAM)

BLOCK ARNOLDI METHOD FOR SYLVESTER-OBSERVER EQUATION

TABLE 4.2

Results for Example 4.2.

(a) Results for Example 4.2 with $A=A_{2}, n=10000, m \in\{3,4,5\}, r \in\{2,5,10\}, c \in\{1,10,30\}$. We used here the restarted shifted FOM(50) to solve the linear systems.

\begin{tabular}{cccccc}
$m$ & $r$ & $c$ & SylvErr & EigErr & $\kappa(X)$ \\
\hline 3 & 2 & 1 & $5.13 \times 10^{-10}$ & $9.10 \times 10^{-10}$ & $1.09 \times 10^{+01}$ \\
5 & 5 & 1 & $3.53 \times 10^{-08}$ & $2.34 \times 10^{-04}$ & $2.28 \times 10^{+00}$ \\
3 & 5 & 10 & $1.32 \times 10^{-12}$ & $1.78 \times 10^{-11}$ & $1.75 \times 10^{+01}$ \\
4 & 5 & 10 & $1.78 \times 10^{-13}$ & $2.72 \times 10^{-11}$ & $4.25 \times 10^{+01}$ \\
4 & 5 & 30 & $9.89 \times 10^{-15}$ & $9.95 \times 10^{-11}$ & $1.21 \times 10^{+03}$ \\
5 & 10 & 30 & $2.38 \times 10^{-13}$ & $2.17 \times 10^{-08}$ & $5.92 \times 10^{+03}$
\end{tabular}

(b) Results for Example 4.2 with $A=A_{3}, n=21341, m \in\{2,3,4\}, r \in\{5,10\}, c \in\{10,30\}$. We used the restarted shifted FOM(50) to solve the linear systems.

\begin{tabular}{cccccc}
$m$ & $r$ & $c$ & SylvErr & EigErr & $\kappa(X)$ \\
\hline 2 & 5 & 10 & $1.04 \times 10^{-10}$ & $8.24 \times 10^{-09}$ & $4.59 \times 10^{+00}$ \\
2 & 5 & 30 & $1.55 \times 10^{-13}$ & $6.65 \times 10^{-12}$ & $4.70 \times 10^{+00}$ \\
3 & 10 & 10 & $4.25 \times 10^{-09}$ & $2.39 \times 10^{-08}$ & $5.17 \times 10^{+00}$ \\
3 & 10 & 30 & $3.24 \times 10^{-10}$ & $3.22 \times 10^{-09}$ & $1.10 \times 10^{+01}$ \\
4 & 5 & 10 & $3.85 \times 10^{-06}$ & $6.41 \times 10^{-05}$ & $5.54 \times 10^{+00}$ \\
4 & 5 & 30 & $2.51 \times 10^{-09}$ & $7.55 \times 10^{-06}$ & $1.73 \times 10^{+01}$
\end{tabular}

(c) Results for Example 4.2 with $A=A_{3}, n=21341, m \in\{2,3,4\}, r \in\{5,10\}, c \in\{10,30\}$. Here Gaussian elimination is used to solve the linear systems.

\begin{tabular}{cccccc}
$m$ & $r$ & $c$ & SylvErr & EigErr & $\kappa(X)$ \\
\hline 2 & 5 & 10 & $1.22 \times 10^{-13}$ & $2.97 \times 10^{-13}$ & $4.59 \times 10^{+00}$ \\
2 & 5 & 30 & $3.25 \times 10^{-14}$ & $1.91 \times 10^{-13}$ & $4.70 \times 10^{+00}$ \\
3 & 10 & 10 & $1.33 \times 10^{-11}$ & $2.39 \times 10^{-09}$ & $5.17 \times 10^{+00}$ \\
3 & 10 & 30 & $1.62 \times 10^{-12}$ & $2.57 \times 10^{-10}$ & $1.10 \times 10^{+01}$ \\
4 & 5 & 10 & $8.92 \times 10^{-09}$ & $1.00 \times 10^{-05}$ & $5.54 \times 10^{+00}$ \\
4 & 5 & 30 & $2.82 \times 10^{-10}$ & $1.56 \times 10^{-07}$ & $1.73 \times 10^{+01}$
\end{tabular}

a prescribed set of $m$ eigenvalues where each eigenvalue is of multiplicity $r$. The $m$ distinct values $\mu_{1}, \ldots, \mu_{m}$ are zeros of the Chebyshev polynomial of the first kind of degree $m$ for the interval $[a+\imath b, a-\imath b]$. The obtained results with the global Arnoldi process are given in Table $4.3 \mathrm{~b}$ while those with the block Arnoldi process are given in Table $4.3 \mathrm{c}$.

We end this last set of experiments by illustrating that the method described in this work allows us to control the multiplicities of the desired eigenvalues. We took $m=3$ and $r=4$ and $\Gamma=\left\{\mu_{1}, \ldots, \mu_{12}\right\}$ where $\mu_{1}=-1, \mu_{2}=-2, \mu_{3}=-3, \mu_{4}=-2, \mu_{5}=-3, \mu_{6}=-4$, $\mu_{7}=-7, \mu_{8}=-6, \mu_{9}=-8, \mu_{10}=-3, \mu_{11}=-4$ and $\mu_{12}=-5$. Table $4.3 \mathrm{~d}$ displays the obtained set of eigenvalues $\sigma(\widehat{H})$ and the $\mu_{k} \mathrm{~s}, k=1, \ldots, m r$ in descending order. For this example, the relative error norms were SylvErr $=7.01 \times 10^{-14}$, EigErr $=3.85 \times 10^{-14}$, and $\kappa(X)=6.8625$.

5. Conclusion. In this paper, we proposed a new method for solving Sylvester-observer equations needed in the construction of the Luenberger observer. This method is a generalization of a single input technique introduced by Datta and Saad and is based on the block Arnoldi process. We gave new algebraic properties and showed how to use the classical block 


\section{ETNA}

Kent State University and

Johann Radon Institute (RICAM)

TABLE 4.3

Results for Example 4.3.

(a) Results for Example 4.3 with $A=A_{4}, n=20000, m \in\{3,5,6,7,8\}, r \in\{3,6,10,20\}$. We used Gaussian elimination to solve the shifted linear systems.

\begin{tabular}{cccccc}
$m$ & $r$ & SylvErr & EigErr & $\kappa(X)$ & time \\
\hline 3 & 10 & $7.94 \times 10^{-14}$ & $3.25 \times 10^{-14}$ & $2.83 \times 10^{+01}$ & 1.31 \\
5 & 3 & $4.12 \times 10^{-13}$ & $1.02 \times 10^{-12}$ & $4.32 \times 10^{+01}$ & 0.62 \\
5 & 6 & $2.33 \times 10^{-13}$ & $8.99 \times 10^{-13}$ & $5.39 \times 10^{+01}$ & 1.42 \\
6 & 20 & $7.77 \times 10^{-13}$ & $1.01 \times 10^{-10}$ & $5.05 \times 10^{+00}$ & 6.00 \\
7 & 20 & $1.36 \times 10^{-12}$ & $5.11 \times 10^{-09}$ & $4.78 \times 10^{+00}$ & 6.82 \\
8 & 10 & $1.59 \times 10^{-11}$ & $6.66 \times 10^{-08}$ & $4.59 \times 10^{+00}$ & 3.87
\end{tabular}

(b) Results for Example 4.3 with $A=A_{4}, n=20000, m \in\{3,5,6,7,8\}, r \in\{3,6,10,20\}$. Gaussian elimination is used to solve the shifted linear systems. The global Arnoldi process was used to solve the Sylvesterobserver equation

\begin{tabular}{cccccc}
$m$ & $r$ & SylvErr & EigErr & $\kappa(X)$ & time \\
\hline 3 & 10 & $6.93 \times 10^{-14}$ & $7.21 \times 10^{-14}$ & $8.49 \times 10^{+00}$ & 0.50 \\
5 & 3 & $4.36 \times 10^{-14}$ & $3.23 \times 10^{-12}$ & $4.48 \times 10^{+00}$ & 0.32 \\
5 & 6 & $1.52 \times 10^{-13}$ & $3.54 \times 10^{-12}$ & $6.68 \times 10^{+00}$ & 0.50 \\
6 & 20 & $1.65 \times 10^{-13}$ & $8.80 \times 10^{-12}$ & $1.55 \times 10^{+01}$ & 1.79 \\
7 & 20 & $1.58 \times 10^{-12}$ & $2.96 \times 10^{-10}$ & $1.70 \times 10^{+01}$ & 2.04 \\
8 & 10 & $2.39 \times 10^{-12}$ & $1.91 \times 10^{-08}$ & $1.16 \times 10^{+01}$ & 1.29
\end{tabular}

(c) Results for Example 4.3 with $A=A_{4}, n=20000, m \in\{3,5,6,7,8\}, r \in\{3,6,10,20\}$. Gaussian elimination was used to solve the shifted linear systems and the block Arnoldi process was used to solve the Sylvesterobserver equation.

\begin{tabular}{cccccc}
$m$ & $r$ & SylvErr & EigErr & $\kappa(X)$ & time \\
\hline 3 & 10 & $4.07 \times 10^{-14}$ & $1.53 \times 10^{-14}$ & $3.83 \times 10^{+01}$ & 0.67 \\
5 & 3 & $3.19 \times 10^{-13}$ & $2.40 \times 10^{-12}$ & $4.85 \times 10^{+00}$ & 0.42 \\
5 & 6 & $3.91 \times 10^{-13}$ & $2.74 \times 10^{-12}$ & $6.69 \times 10^{+00}$ & 0.68 \\
6 & 20 & $2.74 \times 10^{-13}$ & $1.57 \times 10^{-11}$ & $7.71 \times 10^{+00}$ & 3.01 \\
7 & 20 & $1.65 \times 10^{-12}$ & $5.34 \times 10^{-10}$ & $6.92 \times 10^{+00}$ & 3.67 \\
8 & 10 & $7.69 \times 10^{-12}$ & $3.59 \times 10^{-08}$ & $4.74 \times 10^{+00}$ & 2.14
\end{tabular}

(d) Results for Example 4.3: the obtained set of eigenvalues $\sigma(\widehat{H})$ and the $\mu_{k} s$. The matrix is $A=A_{4}, n=20000$, $m=3, r=4$ and the Gaussian elimination was used to solve the shifted linear systems.

\begin{tabular}{cc}
$\Gamma$ & $\sigma(\widehat{H})$ \\
\hline$-1.000000000000000 \mathrm{e}+00$ & $-1.000000000000000 \mathrm{e}+00$ \\
$-2.000000000000000 \mathrm{e}+00$ & $-1.999999999999913 \mathrm{e}+00$ \\
$-2.000000000000000 \mathrm{e}+00$ & $-1.999999999999990 \mathrm{e}+00$ \\
$-3.000000000000000 \mathrm{e}+00$ & $-2.999999999999960 \mathrm{e}+00$ \\
$-3.000000000000000 \mathrm{e}+00$ & $-2.999999999999990 \mathrm{e}+00$ \\
$-3.000000000000000 \mathrm{e}+00$ & $-3.000000000000323 \mathrm{e}+00$ \\
$-4.000000000000000 \mathrm{e}+00$ & $-3.999999999999596 \mathrm{e}+00$ \\
$-4.000000000000000 \mathrm{e}+00$ & $-4.000000000000048 \mathrm{e}+00$ \\
$-5.000000000000000 \mathrm{e}+00$ & $-5.000000000000042 \mathrm{e}+00$ \\
$-6.000000000000000 \mathrm{e}+00$ & $-5.999999999999949 \mathrm{e}+00$ \\
$-7.000000000000000 \mathrm{e}+00$ & $-7.000000000000256 \mathrm{e}+00$ \\
$-8.000000000000000 \mathrm{e}+00$ & $-7.999999999999893 \mathrm{e}+00$
\end{tabular}


Arnoldi algorithm to build the Luenberger observer. The presented numerical tests show that the proposed approach can be quite effective for large-scale Luenberger observer problems.

Acknowledgments. The authors would like to thank the anonymous referees for their numerous and valuable suggestions which improved this paper. Many thanks also to Lothar Reichel, Yousef Saad, Valeria Simoncini, and Paul Van Dooren for discussions and comments on an early version of this work.

\section{REFERENCES}

[1] A. C. Antoulas, Approximation of Large-Scale Dynamical Systems, SIAM, Philadelphia, 2005.

[2] C. Bischof, B. N. DATTA, AND A. PURKYASTHA, A parallel algorithm for the Sylvester-observer matrix equation, SIAM J. Sci. Comput., 17 (1996), pp. 686-698.

[3] D. Calvetti, B. Lewis, AND L. Reichel, On the solution of large Sylvester-observer equations, Numer. Linear Algebra Appl., 8 (2001), pp. 1-16.

[4] J. CARVAlho, K. DatTA, AND Y. Hong, A new block algorithm for full-rank solution of the Sylvesterobserver equation, IEEE Trans. Automat. Control, 48 (2003), pp. 2223-2228.

[5] B. N. DatTA, Numerical Methods for Linear Control Systems Design and Analysis, Academic Press, San Diego, 2004.

[6] B. N. DatTA, M. Heyouni, AND K. JBILou, The global Arnoldi process for solving the Sylvester-observer equation, Comput. Appl. Math., 29 (2010), pp. 527-544.

[7] B. N. DATTA AND Y. SAAD, Arnoldi methods for large Sylvester-like observer matrix equations, and an associated algorithm for partial spectrum assignment, Linear Algebra Appl., 154/156 (1991), pp. 225244.

[8] B. N. DATTA AND D. SARKISSIAN, Block algorithms for state estimation and functional observers, in Proceedings of the IEEE International Symposium on Computer-Aided Control System Design, 2000, IEEE Conference Proceedings, Los Alamitos, 2002, pp. 19-23.

[9] L. ElbouyahyaOUI, A. MessaOUdi, AND H. SADOK, Algebraic properties of the block GMRES and block Arnoldi methods, Electron. Trans. Numer. Anal., 33 (2009), pp. 207-220.

http://etna.ricam.oeaw.ac.at/vol.33.2008-2009/pp207-220.dir/ pp207-220.pdf

[10] A. El Guennouni, K. JBILOU, AND J. RiQuet, Block Krylov subspace methods for solving large Sylvester equations, Numer. Algorithms, 29 (2002), pp. 75-96.

[11] A. El Guennouni, K. JBILOU, AND H. SADOK, The block Lanczos method for linear systems with multiple right-hand sides, Appl. Numer. Math., 51 (2004), pp. 243-256.

[12] A. Frommer, BiCGStab(l) for families of shifted linear systems, Computing, 70 (2003), pp. 87-109.

[13] A. Frommer AND U. GLÄSSNER, Restarted GMRES for shifted linear systems, SIAM J. Sci. Comput., 19 (1998), pp. 5-26.

[14] N. J. Higham, Algorithm 694: a collection of test matrices in MATLAB, ACM Trans. Math. Software, 17 (1991), pp. 289-305.

[15] K. JBILOU, A. MeSSAOUdi, AND H. SADOK, Global FOM and GMRES algorithms for matrix equations, Appl. Numer. Math., 31 (1999), pp. 49-63.

[16] T. KaILATH, Linear Systems, Prentice-Hall, Englewood Cliffs, 1980.

[17] M. Kent, Chebyshev, Krylov, Lanczos: Matrix Relationships and Computations, PhD. Thesis, Department of Computer Science, Stanford University, Stanford, 1989.

[18] D. G. LUENBERGER, Observers for multivariable systems, IEEE Trans. Automat. Control, 11 (1966), pp. 190197.

[19] Y. SAAD, Iterative Methods for Sparse Linear Systems, PWS, Boston, 1996.

[20] B. SHAFAI AND S. P. BHATTACHARYYA, An algorithm for pole assignment in high order multivariable systems, IEEE Trans. Automat. Control, 33 (1988), pp. 870-876.

[21] V. Simoncini, Ritz and pseudo-Ritz values using matrix polynomials, Linear Algebra Appl., 241/243 (1996), pp. 787-801.

[22] - Restarted full orthogonalisation method for shifted linear systems, BIT, 43 (2003), pp. 459-466.

[23] V. SimONCINI AND E. GALlOPOULOS, Convergence properties of block GMRES and matrix polynomials, Linear Algebra Appl., 247 (1996), pp. 97-119.

[24] K. M. SOODhalter, D. B. SZYLD, AND F. XUE, Krylov subspace recycling for sequences of shifted linear systems, Appl. Numer. Math., 81 (2014), pp. 105-118.

[25] P. VAN DoOREN, Reduced order observers: a new algorithm and proof, Systems Control Lett., 4 (1984), pp. 243-251. 
ETNA

Kent State University and

Johann Radon Institute (RICAM)

[26] G. WU, Y. WANG, AND X. JIN, A preconditioned and shifted GMRES algorithm for the PageRank problem with multiple damping factors, SIAM J. Sci. Comput., 34 (2012), pp. A2558-A2575. 